\title{
ABSTRACTS OF CURRENT LITERATURE.
}

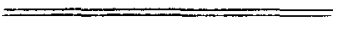

MEDICINE.

\section{GENERAL MEDICINE.}

LHERMitTe: The Syndrome of Quadrigeminal Neoplasms: Relation of Tumours to the Corpora Quadrigemina to Trauma. "Gazette des Höpitaux." January, 1920.

In spite of assiduous research, Ihermitte states that the functions of the corpora quadrigemina remain in obscurity. On experiments on the monkey, partial destruction of the corpora quadrigemina does not lead to any permanent disability-any persisting disorder being due rather to injury of subjacent nerve bundles, Our knowledge of the normal and pathological anatomy of these bodies is limited to their connections with the motor nuclei of the eye, the superior cerebellar bundles, the posterior longitudinal bundle, the paths of general sensibility, the acoustic sense, and finally with the pyramidal bundles.

A destructive lesion-hæmorrhage or sofiening, or a neoplasmgumma or tuberculoma, bring about functional or organic lesions of bundles which mask purely quadrigeminal symptoms. Lhermitte seeks, therefore, not to define the syndrome but to arrive at a possible method of diagnosis of neoplasms of the corpora quadrigemina.

Jhermitte goes on to show the inconsistence of the so-called quadrigeminal syndrome. Nothangel states that lesions of the superior bodies produce disturbances of equilibration and coordination; but pathological investigations do not furnish any proof of this. Lesions of the superior pair lead to diminution of visual acuity or blindness; lesions of the inferior pair lead to a paralysis or paresis of the oculo-motor nerve. One cannot state anything definite on the pupillary condition, but it appears that lesions of the superior pair lead to a loss of pupillary reaction. Nothangel bases his diagnosis on the following symptoms:-(1) 
Titubating gait, identical with that of cerebellar ataxia; (2) Bilateral asymetrical ophthalmoplegia. Weinland and Siebernmann add auditory disturbances to these symptoms. Yet Nothangel's syndrome was not borne out in a case of Nessen's in which autopsy revealed a tumour definitely limited to the corpora quadrigemina, although Nothangel's two pathognomonic signs were absent. Von Minakow is forced to admit that none of these symptoms are characteristic. He says that disturbances of vision are not many; lesions of the superior bodies lead to dilatation of pupils which react imperfectly to light and accommodation, though these even are not constant in experiments on the cat and monkey. Ocular palsies are, according to Von Minakow, more frequent than pupillary changes. Disturbances of co-ordination are frequently observed-sometimes due to true atony or cerebellar inertia. Auditory phenomena-reduced acuity of hearing or subjective noises-are not typical of, though often associated with, lesions of the corpora. Millain also regards Nothangel's two symptoms (ataxia and ophthalmoplegia) as non-pathognomonic of lesions of the corpora.

Lhermitte argues that cases of tumours strictly limited to the quadrigeminal region will alone definitely establish the clinical picture. He cites the following case history :-A soldier, Louis F. (family and personal history negative), was wounded on September 25, 1915, on the left postero-parietal region by a piece of shell; was trephined in October for fracture of the cranial vault, with nervous disturbances and failure of memory. $\mathrm{He}$ was discharged on January 1, 1916, with general asthenia, slight headaches and periodic vomiting. In September, 1916, he rejoined his regiment, all troubles having disappeared. On December 25, 1916, F. displayed noticeable adiposity. Early in 1917, F. had an epileptic fit (general), followed by prolonged coma. In October, 1917, he reported the following symptoms:-Occipital headache, vertigo, failure of memory, drunken gait, disturbances of equilibration with positive Romberg and exaggerated patellar reflexes. Later nausea and vomiting supervened. He could only stand with the legs widely separated; any attempt at bringing the legs together was accompanied by loss of balance. There was incoordination of the lower extremities without tremor, positive Kernig, slight ataxy of the upper extremities. Superficial and deep sensation, and cutaneous reflexes were not disturbed. The reflexes in the upper extremity were increased on the right side; muscular power was retained in the four limbs; muscle tone was increased in the lower limbs. There were noises in the ear, and diminution of auditory power for low sounds. Vision was normal, except for slight myopia, and divergent strabismus dating from infancy. Extrinsic muscles, pupillary reflexes, optic discs, and field of vision were normal. The psychic functions-except memory-were undisturbed. He was forgetful. 
Lumbar puncture yielded a fluid containing no albumen or excess of leucocytes, and under normal pressure.

In April, 1918, the patient had become very fat, with exaggerated appetite and somnolence without polydypsia, polyuria, or glycosuria. Later syncopal attacks with convulsions, and accompanied by dyspncea, cyanosis, and regular, rapid pulse, occurred. Pupils were normal. Babinski appeared bilaterally with nocturnal vomiting. Later convulsions with coma occurred at night time. On April 22nd, 1918, the patient became comatose, with stertorjus breathng. Rhonchi were audible. The patient then became perfectly insensitive, with divergent squint and absence of pupillary reaction. Babinski and Oppenheim phenomena were positive. The tendon reflexes were normal and equal; the pulse full and regular. There was no further convulsion. The patient died without regaining consciousness on April 25, 1918.

Autopsy revealed a depressed fracture about the size of a shilling in the right parietal region. Underneath the dura mater was intact. The inner table was fractured. The pia mater and arachnoid were thickened in the frontal region. No meningeal hæmorrhages. Signs of recent hæmorrhages on the frontal cortex. The basal lepto-meninges were not thickened. Hypophysis normal. Considerable distention of the cerebral ventricles and of the aqueduct of Sylvius. Bulb normal. After hardening in formalin macroscopic section revealed increased size of the quadrigeminal region, and especially increase of the inferior quadrigeminal bodies. These presented a small nodule, about the size of a cherry, situated exactly between the aqueduct of Sylvius and the quadrigeminal group. Histological examination revealed a glioma limited to the ventral portion of the corpora. The superior cerebellar peduncles were compressed and separated from one another by the neoplasm.

Apart from the consequences of the injury to the cranium, Lhermitte considers the results of the glioma infiltrating the corpora quadrigemina. The somnolence, excessive appetite and adiposity, combined with the drunken gait, were most striking. The inco-ordination in the patient presented all the characteristics of cerebellar inco-ordination, except in the fact that the disturbances were much more pronounced in the lower than in the upper extremities. The superior cerebellar peduncles were separated, and compressed by the tumour-accounting for the peculiar locomotion and stance of the patient. There were no choreiform movements or athetosis-an argument against the German hypothesis that these movements are due to a lesion of the superior cerebellar peduncles. The hypoacusis occurring in the patient was due to pressure of the tumour on the path of auditory sensations. The absolute integrity of the visual apparatus is noteworthy in the light of the importance of ocular disturbances insisted on by other authors. There was no disturbance of phonation or deglutition. 


\section{DUBLIN JOURNAL OF MEDICAL SCIENCE}

Thermitte considers the association of disturbances of coordination with auditory manifestations (exclusive of vestibuiar phenomena) as peculiar to a tumour in this situation. The somnolence and disturbances of the general metabolism are relatively frequent in all intracranial tumours-whatever their situation may be.

Lhermitte is finally led to separate the symptoms of quadrigeminal neoplasms into two groups. One group is characterised by a cerebellar type of inco-ordination with hypoacusis, due to pressure on nerve bundles subjacent to the corpora. The other group is characterised by somnolence, adiposity and polyplagia, probably due to ventricular hyper-distension.

Lastly, the author considers the medico-legal question created by the association of quadrigeminal tumours with trauma. Was it coincidence, or was the trauma an æetiological factor in the creation of the quadrigeminal tumour? Examples are cited to show the relationship of trauma to gliomata in this neighbourhood. Grave concussion is felt with maximum intensity in the region of the cerebral ventricles-especially in the third and fourth. Ihermitte definitely believes in the relationship betweel. trauma and gliomata in the region of the corpora quadrigemina.

J. B. MagbnNis.

SöDerbergh: Studies in the Neurology of the Abdominal Irall with regard to Wuscular and Reflex Segmentation. "Acta Medica Scandinavica." Vol. III. Fasc. V.

IN this work, Söderbergh gives a synthesis of observations on the question of the innervation of the abdominal muscles and of abdominal reflexes. He states the effects of electrical stimulation of the 5 th, 6 th, 7 th, 8 th, and 10 th dorsal motor roots. His experiments go to prove that the abdominal muscles are innervated by the motor roots in an entirely segmentary manner; and he controverts Oppenheim's opinion that each segment of the muscles is innervated by all the lower five dorsal segments. The segmentation is not so simple that each muscle segment is provided for by a single root: several contribute, but propably there are one or two principal roots.

The author then draws attention to the motor syndromes of the abdomen. He reports cases to estabiish clinically the motor symptoms limited to different regions of the abdomen, and distinguishes three syndromes. One case of multiple medullary tumours (15)-all operated-was so exceptional in its evolution that he was able to compare the different clinical pictures of the root or medullary lesions. The upper syndrome consists of a lesion of the 7 th dorsal, the middle syndrome of a lesion of the 8 th and 9 th dorsal, and the lower syndrome of a lesion of the 10th dorsal. The 


\section{ABSTRACTS OF CURRENT LITERATURE 253}

upper syndrome comprised motor symptoms of the upper third of the lateral wall of the abdomen, and of the rectus above the umbilicus; the middle syndrome comprised motor symptoms of practically the middle third of the lateral wall of the abdomen; the lower syndrome comprised motor symptoms of the lower region of the abdomen, and of the rectus below the umbilicus. The author goes on to describe cases of cord tumours, discussing electrical reactions obtained, and operative and autopsy findings. He discusses the musculo-spinal segmentation of the abdomen, and the effects of irritation of the motor roots. He lays stress on the point that in cases of localised muscular defence, the patient contracts his muscles not so much to protect himself from the pain of palpation, but rather as the result of a kind of reflex. It is a sub-conscious act; we cannot spontaneously produce partial contractions of the abdominal wall. These partial contractions are only understandable on the supposition of a musculo-spinal segmentation. He suggests detailed research to compare the distribution of peritoneal lesions found at operation, with the extent of the defence. We depend too much on disturbances of sensation for the localisation of tumour levels, and not enough on motor and reflex signs, which are more objective than sensory disturbances. The majority of extra-medullary tumours are in the dorsal region. Should we not, therefore, endeavour to make a more accurate diagnosis of the level of the tumour? Semiology is the foundation on which the edifice should be built, when it is a question of making a precise diagnosis.

J. B. MaGennis

Vines, H. W. C. : Anaphylaxis in the Treatment of Hixmophilia. "Quar. Jour. of Med." April, 1920. P. 257.

Thts paper describes a method of treatment which was found satisfactory in two cases of severe hæmorrhage in hæmophilies.

The first patient, whose personal and family history were characteristic, was admitted to hospital suffering from uncontrolled bleeding from a cut over one eye. 'I'en c.cs. of normal horse serum, given subcutaneously, produced a temporary shortening of the coagulation time, but this was soon followed by a delay in coagulation. Hæmorrhage, which had been arrested by stitching and tight bandaging, recommenced ten days later. It was feared that a further injection of horse serum might produce anaphylaxis, so, in order to test this, two minims of horse serum were given intradermically. A well-marked local reaction with no apparent general response followed; but no further hæmorrhage occurred from the wound. When the coagulation time was tested it was found to be about normal, and remained so for twelva days. Subsequent intradermic injections produced less and less 


\section{DUBLIN JOURNAL OF MEDICAL SCIENCE}

local reaction and less marked acceleration of the coagulation time, till finally such injections were without effect, and the time of coagulation was again very much delayed. It was concluded that the patient was no longer anaphylactic to horse serum, and it was decided to sensitise him to sheep serum. Three c.cs. of this were given subcutaneously, after which the coagulation time fluctuated considerably. On the twelfth day an intradermic injection of sheep serum gave rise to a very marked local reaction. The coagulation time fell rapidly to normal, and remained so until the patient was discharged.

A second patient, also with a typical hæmophilic history, was admitted with uncontrolled hæmorrhage from his lip. Ten c.cs. of normal horse serum were given subcutaneously. The coagulation time remained prolonged until the eleventh day, when twofifths of a minim of serum were given intradermically. The coagulation time fell to normal within twenty-four hours, and remained about this level until the patient was discharged, eight days later.

This method of treatment would appear to be worthy of a further test in the hands of those who are called upon to deal with dangerous hæmorrhage in hæmophilics. It does not interfere with the usual method of treatment; indeed, the intradermic injection is necessary in those cases which have been previously given serum subcutaneously in order to test if a further large injection would be safe.

The method does not promise a cure for hæmophilia, but it offers some hope of tiding the patient over a dangerous period when continued hæmorrhage threatens life.

J. W. BIGGER.

McCaskey, G. W. : Basul Metabolism Determinations in General Internal Diugnosis. "Jour. Amer. Med. Assoc." Vol. 74. P. 927. April 3, 1920.

McCaskey holds that the basal metabolism test rests on an established scientific basis, and that the Benedict method places it on a practical clinical basis. While this method of investigation involves more than the thyroid problem, yet he thinks that the one important controlling factor is thyroxin, on which the functional activity of the thyroid gland is largely, if not altogether, dependent. The question under investigation is the dependability of fluctuation of the basal metabolic rate as an indication of thyroid activity. This investigator considers that routine determinations of basal metabolism are worth while in the following groups of cases :-(I) All cases of definite goitre, especially if associated with health disturbances; (2) cases with or without goitre, with symptoms resembling closely or remotely those of thyrotoxicosis. The estimation of the toxicity of a goitre as determined by local metabolism is of great importance in considering the 
necessity for, and character of, therapeutic measures or in judging of operative risk in thyroidectomy, for this latter increases to an appalling degree when the metabolic rate attains an increase of around 100 per cent. Further, this author is of the opinion that most reliable proof of thyrotoxicosis is an increase in the basal metabolism test. Several cases illustrative of the value of the basal metabolism test in patients suspected of abnormalities of the thyroid internal secretion are given. So far as McCaskey is concerned, the test has passed the experimental stage, and has been assigned a place in his diagnostic armamentarium, although he admits that its precise limitations and scope will only become more closely defined with larger experience.

H. Moone.

Woodburx, M. S. : A Comparison of Methods for Jetermining Thyrotoxicosis. “Jour. Amer. Med. Assoc." Vol. 74. P. 997. April 10, 1920.

THE observations made by Woodbury in this study were prompted by a desire to compare the epinephrin chloride test as described by Getsch, with basal metabolism determinations as advocated by Benedict and Du Bois, in relation to the clinical condition of the patient, the pathologic anatomy of the thyroid after operative removal, and the clinical result of operation. Considering the wide acceptance of increase in the basal metabolism rate as, under the usual conditions, meaning thyrotoxicosis, it seems a matter of importance to discover whether or not so general a conclusion is justified. It is Woodbury's opinion that there is a large group of patients who are in an impaired physical and nervous condition, and who, because of a toxic state of probable thyroid origin, are in need of treatment, and yet whose basal metabolism rate is not increased. It is these latter cases particularly with which the study deals. Excluding five active exophthalmic cases and three markedly toxic adenomata, all of which showed increased metabolism rates of from 20 per cent. to 85 per cent. above normal, there rensain 19 non-exophthalnic adenomatous cases in whom operation was performed, and who were thought to. be suffering from varying degrees of thyrotoxicosis; none of these 19 cases showed increase in the metabolic rate. Of these 19 cases 11 are available for analysis; these 11 cases appear to be definitely thyrotoxic: they all gave a positive epinephrin chloride test, were definitely benefited, with one exception, by operation, gave thyroid glands which, on pathological examination, appeared to be compatible with the diagnosis of thyrotoxicosis, and yet showed basal metabolism rates within normal limits. He concludes that observation of the rate of basal metabolism alone should not be regarded as a sufficient criterion in determining the presence or absence of toxic states associated with the thyroid. 
Although the evidence thus obtained is of much value in estimating varying degrees of toxicity, it should receive only proper emphasis, the complete study of the patient furnishing the only safe guide. The epinephrin chloride test is more sensitive than that of the basal metabolism rate, but requires even greater safeguards than the latter.

H. Moone.

Stokes, J. H., and Busman, G. J. : Tubing as " C'ause of Reaction to Intratenous Injection, esperiully of Assphenamin. "Jour. Amer. Med. Assoc," Vol. 74. P. 117. April 10, 1920.

The authors for the past two years have been investigating the causes of an arsphenamin reaction observed in their clinic. The reaction was characterised by chill, rise of temperature, nausea, vomiting, diarrhoea, pain in the back and head, and prostration. The reaction would appear in crops, so to speak, and disappear for a considerable period. After much investigation the following conclusions were arrived at:-(1) A certain widely distributed brand of so-called pure gum rubber tubing seems to contain when new, a toxic agent responsible for a definite type of reaction following intravenous administration of arsphenamin, and possibly of alkaline solutions in transfusion media; (2) the toxic substance. gradually disappears in the course of a few days from the tubing with use, and is apparently removable in the first instance by soaking the tube for six hours in normal sodium hydroxide solution and rinsing; (3) the toxic property is not destroyed by boiling for one hour, is not soluble in water, and is transmitted to solutions of arsphemamin, neo-arsphenamin and sodium hydroxide merely on passing them through a new tube en route from container to vein.

H. Moonk. 